\title{
Comparison between myocardial function assessed by echocardiography during hospitalization for COVID-19 and at 4 months follow-up
}

\author{
F. M. A. van den Heuvel ${ }^{1}$ (1) - J. L. $\operatorname{Vos}^{1}$ - B. van Bakel ${ }^{2}$ - A. L. Duijnhouwer ${ }^{1}$ - A. P. J. van Dijk ${ }^{1}$ - A. C. Dimitriu-Leen ${ }^{1}$. \\ P. C. Koopmans ${ }^{3} \cdot$ Q. de Mast ${ }^{4} \cdot$ F. L. van de Veerdonk ${ }^{4} \cdot$ F. H. Bosch ${ }^{5} \cdot$ B. van den Borst ${ }^{6} \cdot$ T. M. H. Eijsvogels ${ }^{2}$. \\ R. R. J. van Kimmenade ${ }^{1}$ R. Nijveldt ${ }^{1}$
}

Received: 26 May 2021 / Accepted: 9 July 2021 / Published online: 20 July 2021

(c) The Author(s) 2021

\begin{abstract}
In patients hospitalized for corona virus infectious disease 19 (COVID-19) it is currently unknown whether myocardial function changes after recovery and whether this is related to elevated cardiac biomarkers. In this single center, prospective cohort study we consecutively enrolled hospitalized COVID-19 patients between 1 April and 12 May 2020. All patients underwent transthoracic echocardiography (TTE) evaluation during hospitalization and at a median of 131 days (IQR; 116-136) follow-up. Of the 51 patients included at baseline, 40 (age: 62 years (IQR; 54-68), 78\% male) were available for follow-up TTE. At baseline, $68 \%$ of the patients had a normal TTE, regarding left ventricular (LV) and right ventricular (RV) volumes and function, compared to $83 \%$ at follow-up $(\mathrm{p}=0.07)$. Median LV ejection fraction $(60 \%$ vs. $58 \%, \mathrm{p}=0.54)$ and tricuspid annular plane systolic excursion ( 23 vs $22 \mathrm{~mm}, \mathrm{p}=0.18$ ) were comparable between hospitalization and follow-up, but a significantly lower RV diameter ( 39 vs. $34 \mathrm{~mm}, \mathrm{p}=0.002$ ) and trend towards better global longitudinal strain (GLS) $(-18.5 \%$ vs $-19.1 \%, \mathrm{p}=0.07)$ was found at follow-up. Subgroup analysis showed no relation between patients with and without elevated TroponinT and/or NT-proBNP during hospitalization and myocardial function at follow-up. Although there were no significant differences in individual myocardial function parameters at 4 months follow-up compared to hospitalisation for COVID-19, there was an overall trend towards normalization in myocardial function, predominantly due to a higher rate of normal GLS at follow-up.
\end{abstract}

Keywords COVID-19 $\cdot$ SARS-CoV-2 $\cdot$ Echocardiography $\cdot$ Myocardial function

R. Nijveldt

robin@ nijveldt.net

1 Department of Cardiology, Radboud University Medical Center, Geert Grooteplein Zuid 10, 6525 GA Nijmegen, The Netherlands

2 Department of Physiology, Radboud Institute for Health Sciences, Radboud University Medical Center, Nijmegen, The Netherlands

3 Section Biostatistics, Department of Health Evidence, Radboud University Medical Center, Nijmegen, The Netherlands

4 Department of Internal Medicine and Radboud Center for Infectious Diseases, Radboud University Medical Center, Nijmegen, The Netherlands

5 Department of Internal Medicine, Radboud University Medical Center, Nijmegen, The Netherlands

6 Department of Pulmonology, Radboud University Medical Center, Nijmegen, The Netherlands

\section{What is new?}

- In recovered COVID-19 patients there is a trend towards better global longitudinal strain and the combination of echocardiographic parameters for LV and RV volumes and function 4 months after hospitalization.

- Elevated TroponinT and/or NT-proBNP during hospitalization was not associated with myocardial function at follow-up.

- Most recovered COVID-19 patients have normal myocardial function on echocardiography at 4 months follow-up (83\%). 


\section{Introduction}

Corona virus infectious disease 19 (COVID-19) has a huge impact on the global healthcare system. A substantial percentage of COVID-19 patients requires hospitalization for supplemental oxygen or invasive ventilation [1]. In hospitalized COVID-19 patients, the reported cardiovascular complications are predominantly acute myocardial injury $[2,3]$, venous thrombo-embolic events $[4$, 5] and arrhythmia [5]. Myocardial function assessed by echocardiography, indicated that left ventricular (LV) systolic dysfunction occurred in 10-27\%, and right ventricular (RV) systolic dysfunction in 10-39\% of non-selected hospitalized COVID-19 patients [6, 7]. In hospitalized COVID-19 patients both structural cardiac abnormalities $[8,9]$ and acute myocardial injury, defined as elevated TroponinT [10], are linked to in-hospital mortality [2, 3]. However, elevated cardiac biomarkers TroponinT and NT-proBNP do not seem to be related to myocardial dysfunction in hospitalized COVID-19 patients [6]. It is currently unknown whether myocardial function changes over time in recovered COVID-19 patients and whether this is related to elevated cardiac biomarkers during hospitalization. Therefore, the aim of this study is to evaluate longitudinal changes in myocardial function in patients hospitalized with COVID-19 compared to 4 months after discharge. Additionally, the relation between elevated cardiac biomarkers during hospitalization and myocardial function at follow-up was evaluated.

\section{Methods}

\section{Patient population}

In this single center prospective cohort study, we recruited non-selected, previously hospitalized COVID-19 patients [6]. In short, non-selected and consecutively admitted patients hospitalized at the COVID-19 nursing ward of the Radboudumc (Nijmegen, the Netherlands) were included between 1 April and 12 May, 2020. Polymerase chain reaction (PCR) testing of a nasopharyngeal sample and a non-enhanced low dose CT thorax was performed to confirm the diagnosis of COVID-19. Further information about in- and exclusion criteria were described previously [6]. Patient data, including demographics, medical history, diagnostics, laboratory examinations, treatment, cardiovascular complications and outcomes were collected and analyzed. Follow-up data were derived from our local electronic health record system. All patients received an online questionnaire for assessing self-reported new and persisting symptoms after COVID-19, medical procedures/ surgery, out-patient visits and rehospitalization. The study protocol was approved by the local medical ethics committee (nr. 2020-6765) and written informed consent was obtained from all patients.

\section{Echocardiographic assessment}

All patients underwent a first clinical transthoracic echocardiogram (TTE) at the COVID-19 nursing ward, in supine position, and a second follow-up TTE (median 131 days, IQR; 116-136) afterwards at the outpatient clinic. TTE was focused on LV- and RV systolic function, LV diastolic function and global longitudinal strain (GLS). We did not extensively assess valvular function. All TTE's were performed by experienced sonographers using one single ultrasound system (Affiniti 70 General, Philips Healthcare, Best, the Netherlands) in hospitalized patients, and another single ultrasound system (EPIQ 7, Philips Healthcare, Best, the Netherlands) at the outpatient clinic. Offline analysis was performed by one single investigator (EACVI TTE certified) using dedicated software (AGFA Enterprise Imaging Cardiology version 8.1.2, AGFA HealthCare, Mortsel, Belgium). Detailed information about echocardiographic assessment was described previously [6]. In short, LV function was assessed with LV ejection fraction (LVEF), GLS and the ratio between early mitral inflow velocity and mitral annular early diastolic velocity (E/e'). GLS was measured using speckle tracking echocardiography on a three beats acquisition from three apical long-axis views with a frame rate $>60$ frames/s. The average three plane GLS was used. LV dysfunction was defined as LVEF below 52\% [11] and/ or GLS worse than -18\% [12] and/or E/e' > ratio 14. RV function was assessed with tricuspid annular plane systolic excursion (TAPSE) and RV systolic excursion velocity (RV $\left.\mathrm{S}^{\prime}\right)$. RV dysfunction was defined as a TAPSE $<17 \mathrm{~mm}$ [11] and/or a RV S' $<10 \mathrm{~cm} / \mathrm{s}$ [11]. For measuring LVEF, preferably a triplane LVEF was used. In case of poor image quality, Simpson's biplane LVEF was used next, and otherwise eyeball LVEF assessment [11]. The best method for quantifying LVEF of each TTE is used for further analysis. A 3 dimensional (3D) LVEF using Dynamic Heart Model (Philips Healthcare, Best, the Netherlands) was only available at follow-up. We compared LVEF between the two TTE's using the same method for quantifying LVEF preferably with triplane and otherwise with Simpson's biplane. If only eyeball LVEF was available in one of the TTE's, we compared LVEF with the average LVEF assessed by other quantifying methods including 3D LVEF. We only compared eyeball LVEF if this was the best method of assessment in both TTE's. A normal TTE regarding LV and RV volumes and function was defined as: $\mathrm{LVEF} \geq 52 \%$, GLS $\leq-18 \%$, TAPSE $\geq 17 \mathrm{~mm}, \mathrm{RV} \mathrm{S}^{\prime} \geq 10 \mathrm{~cm} / \mathrm{s}, \mathrm{E} / \mathrm{e}^{\prime}$ ratio $\leq 14$, indexed 
LVmass $\leq 115 \mathrm{~g} / \mathrm{m}^{2}$, Indexed LV end diastolic diameter $\leq 31 \mathrm{~mm}$ and $\mathrm{RV}$ basal diameter $\leq 42 \mathrm{~mm}$ [11].

\section{Cardiac biomarkers}

High-sensitive TroponinT and NT-proBNP concentrations (Roche Diagnostics, Penzberg, Germany) were measured during hospitalization, within $48 \mathrm{~h}$ of the TTE. Elevation in TroponinT levels were defined as a value $>14 \mathrm{ng} / \mathrm{L}$ (i.e. 99th percentile of upper reference limit) and elevated NT-proBNP was defined as a value $>300 \mathrm{pg} / \mathrm{mL}$.

\section{Statistical analysis}

Categorical variables are summarized with counts and percentages, continuous variables with median and interquartile range (non-normal distribution). All statistical analyses were performed using IBM SPSS statistics version 25 (Armonk, New York, United States). A p-value of $<0.05$ will be considered significant. The Wilcoxon signed-rank test was used for comparing echocardiographic parameters (non-normal distribution) across timepoints. For categorical variables a McNemar test for paired data is used. Subgroup analyses of echocardiographic parameters at follow-up were performed using the Mann-Whitney U test. The following subgroups were defined: intensive care unit (ICU) admission, pulmonary embolism diagnosed during hospitalization, elevated TroponinT, elevated NT-proBNP and persistent self-reported symptoms.

\section{Results}

\section{Study population}

Fifty-one patients were initially enrolled of which 40 (78\%) were available for follow-up: 5 were excluded because of no consent to participate, 3 because of palliative oncology care, 1 because of large travel distance, 1 because of hospitalization elsewhere and 1 deceased during index hospitalizing. Of the remaining 40 patients, median age was 62 years (IQR; 54-68), 78\% was male, and the median body mass index was $27 \mathrm{~kg} / \mathrm{m}^{2}$ (IQR; 24-29) (Table 1). Twenty five percent had a history with cardiovascular disease including obstructive coronary artery disease $(13 \%)$, myocardial infarction (13\%) and atrial fibrillation (8\%). Hypertension $(40 \%)$ was the most frequent cardiovascular risk factor, followed by diabetes (18\%). Of all patients, $25 \%$ was on immunosuppressive therapy, and $13 \%$ on oral anticoagulation at admission. The majority of patients $(90 \%)$ had a positive COVID-19 PCR test. The remaining 10\% was considered as 'probable COVID-19', based on high clinical suspicion of COVID-19 and CT findings (CO-RADS 4-5), and were
Table 1 Baseline characteristics

\begin{tabular}{|c|c|}
\hline & Patients $(n=40)$ \\
\hline Male & $31(77.5 \%)$ \\
\hline Age (years) & $62.5(53.5-68.0)$ \\
\hline Body mass index $\left(\mathrm{kg} / \mathrm{m}^{2}\right)$ & $27(24-29)$ \\
\hline \multicolumn{2}{|l|}{ Comorbidities } \\
\hline Cardiac history & $\mathrm{n}=10(25 \%)$ \\
\hline Obstructive coronary artery disease & $\mathrm{n}=5(12.5 \%)$ \\
\hline Myocardial infarction & $\mathrm{n}=5(12.5 \%)$ \\
\hline Non-ischemic cardiomyopathy & $\mathrm{n}=0(0 \%)$ \\
\hline Heart failure & $\mathrm{n}=0(0 \%)$ \\
\hline Atrial fibrillation & $\mathrm{n}=3(7.5 \%)$ \\
\hline Ventricular arrythmias & $\mathrm{n}=1(2.5 \%)$ \\
\hline Moderate- to severe valvular disease & $\mathrm{n}=1(2.5 \%)$ \\
\hline Cardiac surgery & $\mathrm{n}=1(2.5 \%)$ \\
\hline Cardiac electronic device & $\mathrm{n}=1(2.5 \%)$ \\
\hline Hypertension & $\mathrm{n}=16(40 \%)$ \\
\hline Diabetes mellitus & $\mathrm{n}=7(17.5 \%)$ \\
\hline Currently smoking & $\mathrm{n}=3(7.5 \%)$ \\
\hline Cerebrovascular disease & $\mathrm{n}=1(2.5 \%)$ \\
\hline Chronic renal failure (GFR $<30$ or dialysis) & $\mathrm{n}=1(2.5 \%)$ \\
\hline Chronic respiratory diseases (COPD/asthma) & $\mathrm{n}=5(12.5 \%)$ \\
\hline \multicolumn{2}{|l|}{ Medication at admission } \\
\hline Immunosuppressive therapy & $\mathrm{n}=10(25 \%)$ \\
\hline Angiotensin converting enzyme inhibitors & $\mathrm{n}=6(15 \%)$ \\
\hline Angiotensin II receptor blockers & $\mathrm{n}=4(10 \%)$ \\
\hline Oral anticoagulation & $\mathrm{n}=5(12.5 \%)$ \\
\hline \multicolumn{2}{|l|}{ Diagnosis of COVID-19 infection } \\
\hline Positive PCR test & $\mathrm{n}=36(90 \%)$ \\
\hline CT-scan performed & $\mathrm{n}=38(95 \%)$ \\
\hline CO-RADS classification based on the CT-scan & $\mathrm{n}=36(90 \%)$ \\
\hline CO-RADS 1 & $\mathrm{n}=0(0 \%)$ \\
\hline CO-RADS 2 & $\mathrm{n}=0(0 \%)$ \\
\hline CO-RADS 3 & $\mathrm{n}=1(2.5 \%)$ \\
\hline CO-RADS 4 & $\mathrm{n}=3(7.5 \%)$ \\
\hline CO-RADS 5 & $\mathrm{n}=22(55 \%)$ \\
\hline CO-RADS 6 & $\mathrm{n}=10(25 \%)$ \\
\hline CT severity score & $13(10.5-16.5)$ \\
\hline \multicolumn{2}{|l|}{ Laboratory findings at admission, median (IQR) } \\
\hline Hemoglobin (mmol/L) & $8.4(7.7-9.0)$ \\
\hline Leucocytes $\left(10^{9} / \mathrm{L}\right)$ & $6.8(4.9-9.7)$ \\
\hline C-reactive protein $(\mathrm{mg} / \mathrm{L})$ & $88(48-165)$ \\
\hline D-dimer (ng/mL) & $1880(505-2715)$ \\
\hline Procalcitonin $(\mu \mathrm{g} / \mathrm{L})$ & $0.20(0.09-1.08)$ \\
\hline GFR $\left(\mathrm{mL} / \mathrm{min} / \mathrm{kg} / \mathrm{m}^{2}\right)$ & $81(66-90)$ \\
\hline $\mathrm{pH}$ & $7.47(7.44-7.49)$ \\
\hline Lactate $(\mathrm{mmol} / \mathrm{L})$ & $1.3(1.2-1.8)$ \\
\hline TroponinT at any timepoint & $\mathrm{n}=37(92.5 \%)$ \\
\hline Troponin (median ng/L) & $12(8-19)$ \\
\hline Troponin $>14 \mathrm{ng} / \mathrm{L}$ at any time point & $\mathrm{n}=19(47.5 \%)$ \\
\hline Troponin $>50 \mathrm{ng} / \mathrm{L}$ at any time point & $\mathrm{n}=3(7.5 \%)$ \\
\hline Troponin $>140 \mathrm{ng} / \mathrm{L}$ at any time point & $\mathrm{n}=1(2.5 \%)$ \\
\hline
\end{tabular}


Table 1 (continued)

\begin{tabular}{ll}
\hline & Patients $(\mathrm{n}=40)$ \\
\hline NT-proBNP at any timepoint & $\mathrm{n}=38(95 \%)$ \\
NT-proBNP (median pg/mL) & $315(94-695)$ \\
NT-proBNP $>300 \mathrm{pg} / \mathrm{mL}$ & $\mathrm{n}=20(50 \%)$ \\
NT-proBNP $>1000 \mathrm{pg} / \mathrm{mL}$ & $\mathrm{n}=3(7.5 \%)$ \\
\hline
\end{tabular}

Values are in median and interquartile range, or $\mathrm{n}(\%)$

$C O P D$ chronic obstructive pulmonary disease, CO-RADS COVID-19 Reporting and Data System, $C T$ computed tomography, GFR glomerular filtration rate, $P C R$ polymerase chain reaction

treated for COVID-19. Low dose CT thorax was performed in $90 \%$ with a median CO-RADS score of 5 and severity score of 13. Duration of hospitalization was 9 days (IQR; $7-22)$. Nearly all patients received supplemental oxygen (93\%) and 14 patients (35\%) were admitted to the intensive care unit (ICU), of whom $12(30 \%)$ were intubated and mechanically ventilated mostly in prone position (Table 2 ). Of the initially 51 patients, only 3 patients were transferred to the ICU department after the first echocardiogram, of whom one patient deceased. Sixteen patients came from the ICU department and underwent echocardiography on the COVID-19 nursing ward thereafter.

\section{Echocardiographic findings}

All echocardiographic parameters are summarized in Table 3. Patients underwent transthoracic echocardiography at a median of 4 days (IQR; $2-11$ days) after hospitalization and second TTE at a median of 131 days (IQR; 116-136) afterwards. Comparing TTE parameters at hospitalization and follow-up, there were no differences between LVEF (60 (IQR; 56-60) \% vs. 58 (IQR; 54-60) \%, p=0.54), LV diastolic function (7.3 vs $7.5 \mathrm{E} / \mathrm{e}^{\prime}$ ratio, $\mathrm{p}=0.90$ ), GLS $(-18.5 \%$ vs $-19.1 \%, \mathrm{p}=0.07)$ and TAPSE $(23$ vs $22 \mathrm{~mm}$, $\mathrm{p}=0.18$ ). Fore comparing LVEF a triplane EF was used in 20 patients, biplane EF in 5 patients, eyeball EF to average EF in 11 patients and in 4 patients only eyeball EF was used. RV basal diameter ( 39 vs. $34 \mathrm{~mm}, \mathrm{p}=0.002$ ) and LV mass ( 83 vs. $71 \mathrm{~g} / \mathrm{m}^{2}, \mathrm{p}=0.04$ ) were higher in patients during hospitalization for COVID-19 compared to follow-up. RV S' (14 vs. $13 \mathrm{~cm} / \mathrm{s}, \mathrm{p}=0.01$ ) was different between both TTE's although the absolute value of RV S' at follow-up was in all but 1 patient (3\%) within the normal range. During hospitalization, 13\% had abnormal LVEF, 38\% abnormal GLS, 5\% abnormal TAPSE, 6\% abnormal RV S' and 10\% abnormal RV basal diameter. At follow-up and compared to hospitalization, 5\% had abnormal LVEF $(p=0.25), 18 \%$ abnormal GLS $(p=0.13), 0 \%$ abnormal TAPSE $(p=0.50)$, $3 \%$ abnormal RV S' $(\mathrm{p}=0.50)$ and $3 \%$ abnormal RV basal diameter $(\mathrm{p}=0.50)$. With regard to a combination of $\mathrm{LV}$
Table 2 Treatment and outcome of the patients

Patients $(n=40)$

Treatment

Treatment with nasal canula/face mask

Nasal high flow therapy

Mechanical ventilation, $\mathrm{n}(\%)$

Number of days (IQR)

Prone ventilation

Medium care unit admission

Intensive care unit admission

Days of admission (IQR)

$$
\begin{aligned}
& \mathrm{n}=37(92.5 \%) \\
& \mathrm{n}=6(15 \%) \\
& \mathrm{n}=12(30 \%) \\
& 16(11-22) \\
& \mathrm{n}=11(27.5 \%) \\
& \mathrm{n}=1(2.5 \%) \\
& \mathrm{n}=14(35 \%) \\
& 14(9-24) \\
& \mathrm{n}=3(7.5 \%) \\
& \mathrm{n}=0(0 \%) \\
& \mathrm{n}=1(2.5 \%) \\
& \mathrm{n}=0(0 \%) \\
& \mathrm{n}=0(0 \%) \\
& \mathrm{n}=3(7.5 \%) \\
& \mathrm{n}=2(5 \%) \\
& \mathrm{n}=7(17.5 \%) \\
& \mathrm{n}=3(7.5 \%)
\end{aligned}
$$

Diagnosed in hospital complications

Acute heart failure

Type 1 myocardial infarction

Type 2 myocardial infarction

Myocarditis

Ventricular arrythmia

Atrial fibrillation

CVA/TIA

Pulmonary embolism

Acute kidney failure

Discharge

Duration of hospital admission (days)

$9(7-22)$

Complications after discharge

Days of follow-up after first TTE (IQR)

Pulmonary embolism

$00(191-206)$

Myocardial infarction

$\mathrm{n}=2(\%)$

$\mathrm{n}=0(0 \%)$

$\mathrm{n}=0(0 \%)$

Acute heart failure

$\mathrm{n}=1(2.5 \%)$

Atrial fibrillation

Myocarditis

Hospitalization for cardiac cause

$\mathrm{n}=0(0 \%)$

$\mathrm{n}=0(0 \%)$

Emergency department visit

$\mathrm{n}=1(2.5 \%)$

Deceased

$\mathrm{n}=0(0 \%)$

Self-reported symptoms after COVID-19*

$\begin{array}{ll}\text { Dyspnea } & \mathrm{n}=11(27.5 \%) \\ \text { Chest pain } & \mathrm{n}=3(7.5 \%) \\ \text { Peripheral edema } & \mathrm{n}=3(7.5 \%) \\ \text { Fatigue } & \mathrm{n}=7(17.5 \%)\end{array}$

Values are in median and interquartile range, or $\mathrm{n}(\%)$

CVA cerebrovascular accident, TIA transient ischaemic attack, TTE transthoracic echocardiogram

*symptoms assessed by online questionnaire at 200 days (IQR; 191206) after TTE

and RV volumes and function parameters $67.5 \%$ of patients had a normal TTE during hospitalization for COVID-19 and $82.5 \%$ at follow-up, with a trend towards statistical significance $(\mathrm{P}=0.07)$. Median LVEF, GLS, TAPSE and RV S' during hospitalization and at follow-up are shown in Fig. 1. Comparison of all cardiac function parameters during hospitalization and at follow-up in each patient are 
Table 3 Echocardiographic parameters of patients

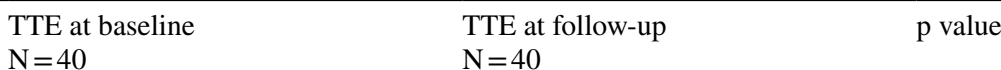

\begin{tabular}{|c|c|c|c|}
\hline \multicolumn{4}{|l|}{ LV dimensions, median (IQR) } \\
\hline Indexed LVEDd $\left(\mathrm{mm} / \mathrm{m}^{2}\right)$ & $24(21$ to 26$)$ & $23(22$ to 26$)$ & 0.976 \\
\hline Indexed LV mass $\left(\mathrm{g} / \mathrm{m}^{2}\right)$ & 83 (68 to 92$)$ & $71(61$ to 95$)$ & 0.036 \\
\hline \multicolumn{4}{|l|}{ LV systolic function median (IQR) } \\
\hline All EF \% $(n=40)$ & $60.0(55.5$ to 60.0$)$ & $58.0(54.3$ to 60.4$)$ & 0.544 \\
\hline Triplane EF-triplane EF $(n=20)$ & $57(52.3$ to 59.0$)$ & $55.0(53.3$ to 58$)$ & 0.793 \\
\hline Biplane EF-biplane EF $(n=5)$ & $63.0(59.5$ to 66.0$)$ & $61.0(58.5$ to 62.0$)$ & 0.221 \\
\hline Eyeball EF-average EF $(n=11)$ & $60.0(60.0$ to 60.0$)$ & $60.0(56.5$ to 63.0$)$ & 0.475 \\
\hline Eyeball EF-eyeball EF $(\mathrm{n}=4)$ & $60.0(60.0$ to 60.0$)$ & $60.0(60.0$ to 60.0$)$ & $>0.99$ \\
\hline $3 \mathrm{D} E F \%(\mathrm{n}=32)$ & - & $61(55.5$ to 64$)$ & - \\
\hline \multirow[t]{2}{*}{ Global longitudinal strain (\%) } & $\mathrm{n}=21$ & $\mathrm{n}=28$ & \\
\hline & $-18.5(-19.5$ to -17.0$)$ & $-19.1(-20.8$ to -18.2$)$ & 0.067 \\
\hline \multicolumn{4}{|l|}{ LV diastolic function median (IQR) } \\
\hline \multirow[t]{2}{*}{$\mathrm{E} / \mathrm{e}^{\prime}$ ratio } & $\mathrm{n}=40$ & $\mathrm{n}=40$ & \\
\hline & $7.3(6.0$ to 9.8$)$ & 7.5 (5.9 to 8.8$)$ & 0.898 \\
\hline \multicolumn{4}{|l|}{ RV dimension and function median (IQR) } \\
\hline \multirow[t]{2}{*}{ RV basal diameter (mm) } & $\mathrm{n}=30$ & $\mathrm{n}=33$ & \\
\hline & $39(33$ to 41$)$ & $34(30$ to 38$)$ & 0.002 \\
\hline \multirow[t]{2}{*}{ TAPSE $(\mathrm{mm})$} & $\mathrm{n}=40$ & $\mathrm{n}=39$ & \\
\hline & 23 (19 to 27$)$ & $22(20$ to 25$)$ & 0.181 \\
\hline \multirow[t]{2}{*}{ RV S' (cm/s) } & $\mathrm{n}=35$ & $\mathrm{n}=38$ & \\
\hline & $14(12$ to 18$)$ & $13(11$ to 15$)$ & 0.01 \\
\hline \multicolumn{4}{|l|}{ Other parameters } \\
\hline Normal LV and RV volumes and function** & $67.5 \%$ & $82.5 \%$ & $0.07 *$ \\
\hline Abnormal LVEF $(<52 \%)$ & $12.5 \%$ & $5.0 \%$ & $0.25^{*}$ \\
\hline Abnormal GLS $(>-18 \%)$ & $38.1 \%$ & $17.9 \%$ & $0.125^{*}$ \\
\hline
\end{tabular}

All values were tested with a Wilcoxon signed-rank test, except for $\left(^{*}\right)$ which was done with a McNemar test. Values are in median and interquartile range, or $\mathrm{n}(\%)$

$E / e^{\prime}$ early mitral inflow velocity/mitral annular early diastolic velocity, $E F$ ejection fraction, $I Q R$ interquartile range, $L V$ left ventricular, $L V E D d$ left ventricular end-diastolic dimension, $L V E S d$ left ventricular end-systolic dimension, $L V E F$ LV ejection fraction, $R V$ right ventricular, $R V S$, right ventricular systolic excursion velocity, TAPSE tricuspid annular plane systolic excursion, TTE transthoracic echocardiogram

$* *$ Normal TTE was defined as: LVEF $\geq 52 \%$, GLS $\leq-18 \%$, TAPSE $\geq 17 \mathrm{~mm}, \mathrm{RV} \mathrm{S}{ }^{\prime} \geq 10 \mathrm{~cm} / \mathrm{s}$, E/e' ratio $\leq 14$, indexed LVmass $\leq 115 \mathrm{~g} / \mathrm{m}^{2}$, Indexed LVEDd $\leq 31 \mathrm{~mm}$ and RV basal diameter $\leq 42 \mathrm{~mm}$

included in the supplemental data. A typical case is shown in Fig. 2. Subgroup analysis revealed no differences regarding myocardial function at follow-up between patients with and without ICU admission or pulmonary embolism diagnosed during hospitalization (Table 4).

\section{Cardiac biomarkers}

At least one TroponinT value was available in $92.5 \%$ of all patients, and $47.5 \%$ had elevated TroponinT during hospitalization. NT-proBNP was measured in $95 \%$ of all patients, of whom 50\% had elevated NT-proBNP during hospitalization. All laboratory values are shown in Table 1. Subgroup analysis showed no differences regarding myocardial function at follow-up between patients with and without elevated TroponinT and/or NT-proBNP (Table 4).

\section{Outcome}

The most frequently diagnosed in-hospital complication was pulmonary embolism (17.5\%) followed by atrial fibrillation (7.5\%), acute heart failure (7.5\%), and acute kidney failure with a glomerular filtration rate below $30 \mathrm{~mL} / \mathrm{min} / 1.73$ $\mathrm{m}^{2}(7.5 \%)$. Follow-up after discharge was available in all patients, with a median duration of 200 days (IQR; 191-206) after the first TTE. After discharge, 2 (5\%) patients had a pulmonary embolism diagnosed on a chest $\mathrm{CT}$ which was performed for other reasons and 1 patient (3\%) developed de novo atrial fibrillation (Table 2). From the online post 



Fig. 1 Boxplot of cardiac function parameters. Boxplot of median and interquartile range of left ventricular ejection fraction (LVEF), global longitudinal strain (GLS), right ventricular systolic excursion

velocity (RV S') and tricuspid annular plane systolic (TAPSE) during hospitalization for corona virus infectious disease 19 (COVID-19) and at follow-up

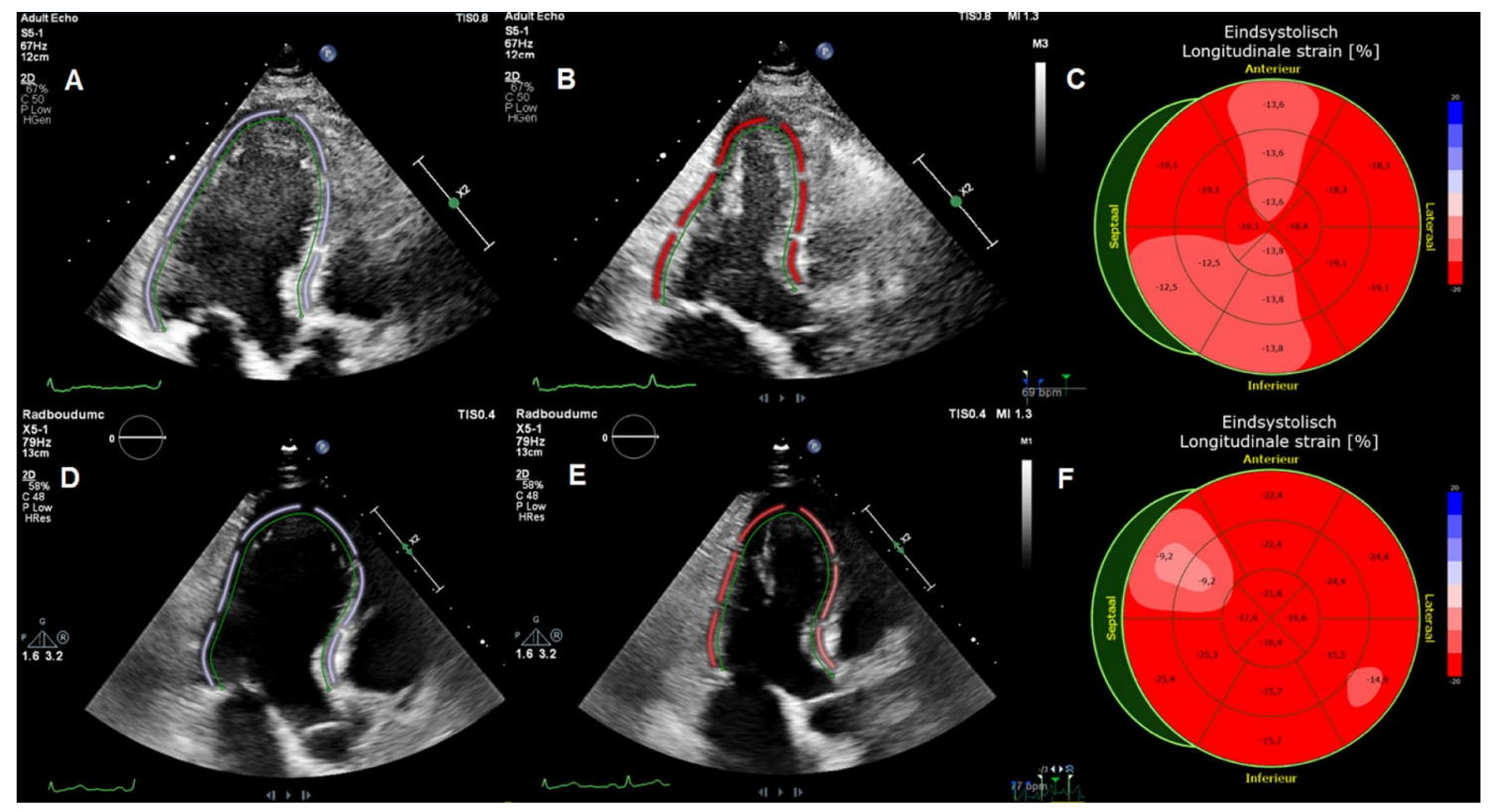

Fig. 2 Typical case. Typical case of a patient with abnormal global longitudinal strain during hospitalization $(-16.1 \%)$ with normal left ventricular ejection fraction $(54 \%)$ and normal global longitudinal strain $(-18.8 \%)$ and left ventricular ejection fraction $(54 \%)$ at 4 months follow-up. A: apical 3 chamber with end diastolic tracing of left ventricle. B: apical 3 chamber with end systolic tracing of left ventricle. C: bull's eye plot of global longitudinal strain. D: apical 3 chamber with end diastolic tracing of left ventricle. E: apical 3 chamber with end systolic tracing of left ventricle. F: bull's eye plot of global longitudinal strain. Image $\mathbf{A}-\mathbf{C}$ are during hospitalization and D-F at 4 months follow-up 
Table 4 Subgroup analysis at follow-up

\begin{tabular}{|c|c|c|c|}
\hline TroponinT & $<14 \mathrm{ng} / \mathrm{l}(\mathrm{n}=18)$ & $>14 \mathrm{ng} / \mathrm{l}(\mathrm{n}=19)$ & $p$ value \\
\hline LVEF \% (median/IQR) & $55.5(54.3$ to 59.8$)$ & $57.0(52.3$ to 61.3$)$ & 0.552 \\
\hline GLS \% (median/IQR) & $-19.1(-20.7$ to -18.1$)$ & $-19.7(-21.4$ to -17.4$)$ & 0.593 \\
\hline TAPSE mm (median/IQR) & $21.0(20.0$ to 23.8$)$ & $23.0(19.5$ to 26.0$)$ & 0.621 \\
\hline RV S' cm/s (median/IQR) & $12.0(10.3$ to 15.5$)$ & $14(11.5$ to 15.5$)$ & 0.525 \\
\hline NT-proBNP & $<300 \mathrm{pg} / \mathrm{mL}(\mathrm{n}=18)$ & $>300 \mathrm{pg} / \mathrm{mL}(\mathrm{n}=20)$ & \\
\hline LVEF \% (median/IQR) & $57.0(54.0$ to 59.5$)$ & $55.5(52.3$ to 60.4$)$ & 0.557 \\
\hline GLS \% (median/IQR) & $-19.7(-21.4$ to -18.2$)$ & $-19.1(-20.7$ to -17.0$)$ & 0.429 \\
\hline TAPSE mm (median/IQR) & $21.0(19.5$ to 26.0$)$ & 22.5 (20.0 to 23.8$)$ & 0.368 \\
\hline RV S' cm/s (median/IQR) & $12.0(11.0$ to 15.0$)$ & $13.0(11.0$ to 16.0$)$ & 0.886 \\
\hline Treatment & No ICU admission $(\mathrm{n}=26)$ & ICU admission $(n=14)$ & \\
\hline LVEF \% (median/IQR) & $56.0(53.5$ to 60.3$)$ & $55.5(52.5$ to 60.3$)$ & 0.659 \\
\hline GLS \% (median/IQR) & $-19.1(-21.1$ to -17.3$)$ & $-19.7(-20.5$ to -18.3$)$ & 0.981 \\
\hline TAPSE mm (median/IQR) & 21.0 ( 20.0 to 24.5$)$ & $23(18.8$ to 26.0$)$ & 0.871 \\
\hline RV S' cm/s (median/IQR) & $12.0(11.0$ to 15.5$)$ & $13.5(10.5$ to 15.8$)$ & 0.783 \\
\hline Complication during hospitalization & No pulmonary embolism $(\mathrm{n}=33)$ & Pulmonary embolism $(n=7)$ & \\
\hline LVEF \% (median/IQR) & $58.0(54.0$ to 60.3$)$ & $58.0(55.0$ to 61.0$)$ & 0.775 \\
\hline GLS \% (median/IQR) & $-19.1(-21.2$ to -18.0$)$ & $-19.4(-20.1$ to -18.6$)$ & 0.921 \\
\hline TAPSE mm (median/IQR) & $22.0(20.0$ to 25.0$)$ & $22.0(20.0$ to 24.0$)$ & 0.941 \\
\hline RV S' cm/s (median/IQR) & $13.0(11.0$ to 15.0$)$ & $13.0(11.0$ to 15.0$)$ & 0.805 \\
\hline $\begin{array}{l}\text { Symptoms (dyspnea/chest pain/fatigue/periph- } \\
\text { eral edema after COVID-19) }\end{array}$ & No symptoms $(n=26)$ & Symptoms $(n=14)$ & \\
\hline LVEF \% (median/IQR) & $56.8(54.0$ to 60.1$)$ & $59.3(56.5$ to 61.9$)$ & 0.151 \\
\hline GLS \% (median/IQR) & $-19.1(-20.4$ to -18.3$)$ & $-19.1(-21.9$ to -17.3$)$ & 0.658 \\
\hline TAPSE mm (median/IQR) & $22.5(20.0$ to 25.3$)$ & $21.0(19.5$ to 25.0$)$ & 0.765 \\
\hline RV S' cm/s (median/IQR) & $13.0(11.0$ to 15.0$)$ & $14.0(10.5$ to 15.0$)$ & 0.938 \\
\hline
\end{tabular}

All values were tested with a Mann-Whitney U test

$G L S$ global longitudinal strain, $L V$ left ventricular, $L V E F$ left ventricular ejection fraction, $R V S$ ' right ventricular systolic excursion velocity, TAPSE tricuspid annular plane systolic excursion

COVID-19 questionnaire, 14 patients (35\%) had selfreported new and/or persisting symptoms after COVID-19. Dyspnea (28\%) was the most frequent symptom, followed by fatigue (18\%), chest pain (8\%), and peripheral edema (8\%). Subgroup analysis revealed no differences regarding myocardial function at follow-up between patients with and without self-reported symptoms (Table 4).

\section{Discussion}

To the best of our knowledge, this is the first study evaluating longitudinal changes in myocardial function in patients with COVID-19 from hospitalization to 4 months after discharge. In this non-selected and consecutively enrolled patient cohort, there was an overall trend towards normalization in myocardial function, predominantly due to a higher rate of normal GLS at follow-up compared to hospitalization. There were no significant changes in LVEF, LV diastolic function and TAPSE over time, but there was a decrease in RV basal diameter. Our findings regarding LV and RV volumes and function during hospitalization for COVID-19 are similar to other echocardiography studies $[7,12,13]$ indicating that the absolute values of LVEF, E/e' ratio, TAPSE, RV S' are often within the normal range while GLS is reduced [12,13] during hospitalization. Furthermore, our results are also in line with cardiovascular magnetic resonance imaging studies [14-16] in recovered COVID-19 patients indicating that LV and RV volumes and function are also most often within the normal range in recovered patients. Although the majority of the echocardiographic findings were within the normal range, we did notice a trend towards completely normal TTE's 4 months after hospitalization, regardless of the biomarkers measured during hospitalization.

Because COVID-19 predominantly affects the respiratory system, theoretically RV function parameters would be expected to show the largest differences between hospitalization and follow-up. The decrease in RV basal diameter at follow-up could hypothetically be due to a decrease or normalization in pulmonary artery pressure 
after pulmonary recovery, however, pulmonary pressures have not been assessed in the current study. RV S' was significantly lower at follow-up compared to hospitalization. We considered the change of RV S' over time as not clinically relevant because the absolute value of RV S' at follow-up remained within the normal range for all but one patient. The most remarkable difference in functional parameters is seen in GLS. GLS during hospitalization is associated with elevated inflammatory markers and hypoxia suggesting that abnormal GLS could be secondary to systemic inflammation [13]. Three months after COVID-19 inflammatory markers are often within the normal range [17]. Although we did not assess inflammatory markers at follow-up, normalization in inflammatory markers could be a potential cause of the trends towards slight improvement in GLS at follow-up compared to hospitalization.

In large international registries, it is estimated that $27.8 \%$ of hospitalized COVID-19 patients require ICU admission [5], 6.6\% have pulmonary embolism [5] and that the incidence of elevated Troponin during hospitalization is $23-36 \%$ [3, 18]. These findings are comparable with our results. Furthermore, in our and other studies $[19,20]$, a significant percentage of recovered COVID-19 patients who required hospitalization have self-reported persisting symptoms such as dyspnea, chest pain, fatigue and/or peripheral edema months after discharge. Because of these in-hospital complications and persisting symptoms, cardiac screening for myocardial dysfunction in recovered COVID-19 patients is a matter of debate. Based on our findings, there is no association between elevated cardiac biomarkers, ICU admission, pulmonary embolism nor persisting symptoms, and myocardial dysfunction at follow-up. Therefore, we do not recommend routine cardiac screening with echocardiography in all recovered previously hospitalized COVID-19 patients. Of note, patients with markedly elevated TroponinT, more than three times the upper reference limit of normal, and/ or NT-proBNP $>1000 \mathrm{pg} / \mathrm{mL}$ during hospitalization are underrepresented in our study and therefore we cannot draw any conclusions from these patients. Further larger scaled studies on cardiac screening and the change in myocardial function in recovered COVID-19 patients are needed.

In conclusion, 4 months after hospitalization for COVID-19, patients had more often a normal TTE than during hospitalization, predominantly related to an increased number of patients with normal GLS at followup. There was no association between elevated cardiac biomarkers during hospitalization and myocardial function at follow-up, nor between symptomatology and echocardiographic functional parameters at follow-up.

\section{Limitations}

Our study has limitations. First, the relatively small sample size, and therefore sub-analyses should be carefully interpreted. We tried to include as many patients as possible during the first wave in the Netherlands but due to a drop of hospitalizations in May 2020, we stopped including. Second, since written informed consent was required, the frailest patients could not be enrolled. Third, TTE's were performed on different ultrasound systems, however, both machines were from the same vendor and analyses were performed using the same software. Fourth, biomarkers were not available at follow-up. Last, we only have follow-up in 40 of the initial 51 patients (78\%) [6] which could lead to a selection bias. However, there were no differences in baseline characteristic such as age, sex, elevated TroponinT and/or elevated NT-proBNP between patients who did and did not participate in the follow-up study (supplementary table).

Supplementary Information The online version contains supplementary material available at https://doi.org/10.1007/s10554-021-02346-5.

Funding Grants: None.

Data availability The data underlying this article will be shared on reasonable request to the corresponding author.

\section{Declarations}

Conflict of interest All authors declare that there is no conflict of interest.

Consent to participate Informed consent was obtained from all patients participants included in the study.

Consent to publish All patients signed informed consent regarding publishing their anonymized data.

Ethical approval The study protocol was approved by the local medical ethics committee (nr. 2020-6765).

Open Access This article is licensed under a Creative Commons Attribution 4.0 International License, which permits use, sharing, adaptation, distribution and reproduction in any medium or format, as long as you give appropriate credit to the original author(s) and the source, provide a link to the Creative Commons licence, and indicate if changes were made. The images or other third party material in this article are included in the article's Creative Commons licence, unless indicated otherwise in a credit line to the material. If material is not included in the article's Creative Commons licence and your intended use is not permitted by statutory regulation or exceeds the permitted use, you will need to obtain permission directly from the copyright holder. To view a copy of this licence, visit http://creativecommons.org/licenses/by/4.0/. 


\section{References}

1. Wiersinga WJ, Rhodes A, Cheng AC, Peacock SJ, Prescott HC (2020) Pathophysiology, transmission, diagnosis, and treatment of coronavirus disease 2019 (COVID-19): a review. JAMA 324(8):782-793

2. Guo T, Fan Y, Chen M, Wu X, Zhang L, He T et al (2020) Cardiovascular implications of fatal outcomes of patients with coronavirus disease 2019 (COVID-19). JAMA Cardiol. https://doi.org/10. 1001/jamacardio.2020.1017

3. Lala A, Johnson KW, Januzzi JL, Russak AJ, Paranjpe I, Richter $\mathrm{F}$ et al (2020) Prevalence and impact of myocardial injury in patients hospitalized with COVID-19 infection. J Am Coll Cardiol 395:497

4. Long B, Brady WJ, Koyfman A, Gottlieb M (2020) Cardiovascular complications in COVID-19. Am J Emerg Med. https://doi. org/10.1016/j.ajem.2020.04.048

5. Linschoten M, Peters S, van Smeden M, Jewbali LS, Schaap J, Siebelink HM et al (2020) Cardiac complications in patients hospitalised with COVID-19. Eur Heart J Acute Cardiovasc Care. https://doi.org/10.1177/2048872620974605

6. van den Heuvel FMA, Vos JL, Koop Y, van Dijk APJ, Duijnhouwer AL, de Mast Q et al (2020) Cardiac function in relation to myocardial injury in hospitalised patients with COVID-19. Neth Heart J 28(7-8):410-417

7. Szekely Y, Lichter Y, Taieb P, Banai A, Hochstadt A, Merdler I et al (2020) The spectrum of cardiac manifestations in coronavirus disease 2019 (COVID-19) - a Systematic Echocardiographic Study. Circulation. https://doi.org/10.1161/CIRCULATIONAHA. 120.047971

8. Giustino G, Croft LB, Stefanini GG, Bragato R, Silbiger JJ, Vicenzi $M$ et al (2020) Characterization of myocardial injury in patients with COVID-19. J Am Coll Cardiol 76(18):2043-2055

9. Rath D, Petersen-Uribe Á, Avdiu A, Witzel K, Jaeger P, Zdanyte $M$ et al (2020) Impaired cardiac function is associated with mortality in patients with acute COVID-19 infection. Clin Res Cardiol 109(12):1491-1499

10. Sandoval Y, Januzzi JL Jr, Jaffe AS (2020) Cardiac Troponin for assessment of myocardial injury in COVID-19: JACC review topic of the week. J Am Coll Cardiol 76(10):1244-1258

11. Lang RM, Badano LP, Mor-Avi V, Afilalo J, Armstrong A, Ernande L et al (2015) Recommendations for cardiac chamber quantification by echocardiography in adults: an update from the American Society of Echocardiography and the European
Association of Cardiovascular Imaging. Eur Heart J Cardiovasc Imaging 16(3):233-270

12. Lassen MCH, Skaarup KG, Lind JN, Alhakak AS, Sengeløv M, Nielsen AB et al (2020) Echocardiographic abnormalities and predictors of mortality in hospitalized COVID-19 patients: the ECHOVID-19 study. ESC Heart Fail 7(6):4189-4197

13. Li R, Wang H, Ma F, Cui GL, Peng LY, Li CZ et al (2021) Widespread myocardial dysfunction in COVID-19 patients detected by myocardial strain imaging using 2-D speckle-tracking echocardiography. Acta Pharmacol Sin. https://doi.org/10.1038/ s41401-020-00595-Z

14. Puntmann VO, Carerj ML, Wieters I, Fahim M, Arendt C, Hoffmann J et al (2020) Outcomes of cardiovascular magnetic resonance imaging in patients recently recovered from coronavirus disease 2019 (COVID-19). JAMA Cardiol 5(11):1265

15. Huang L, Zhao P, Tang D, Zhu T, Han R, Zhan C et al (2020) Cardiac involvement in patients recovered from COVID-2019 identified using magnetic resonance imaging. JACC Cardiovasc Imaging 13(11):2330-2339

16. Rajpal S, Tong MS, Borchers J, Zareba KM, Obarski TP, Simonetti OP et al (2020) Cardiovascular magnetic resonance findings in competitive athletes recovering from COVID-19 infection. JAMA Cardiol. https://doi.org/10.1001/jamacardio.2020.4916

17. van den Borst B, Peters JB, Brink M, Schoon Y, Bleeker-Rovers $\mathrm{CP}$, Schers $\mathrm{H}$ et al (2020) Comprehensive health assessment three months after recovery from acute COVID-19. Clin Infect Dis. https://doi.org/10.1093/cid/ciaa1750

18. Richardson S, Hirsch JS, Narasimhan M, Crawford JM, McGinn T, Davidson KW et al (2020) Presenting characteristics, comorbidities, and outcomes among 5700 patients hospitalized with COVID-19 in the New York City area. JAMA. https://doi.org/10. 1001/jama.2020.6775

19. Carfî A, Bernabei R, Landi F (2020) Persistent symptoms in patients after acute COVID-19. JAMA 324(6):603-605

20. Garrigues E, Janvier P, Kherabi Y, Le Bot A, Hamon A, Gouze $\mathrm{H}$ et al (2020) Post-discharge persistent symptoms and healthrelated quality of life after hospitalization for COVID-19. J Infect 81(6):e4-e6

Publisher's Note Springer Nature remains neutral with regard to jurisdictional claims in published maps and institutional affiliations. 